\title{
MS13-P01 | BRACHIOPOD SHELL CALCITE MINERALIZATION OCCURS BY ION-BY-ION TRANSPORT CONTROLLED BY THE EPITHELIAL CELL MEMBRANES
}

Griesshaber, Erika (LMU Munich, Munich, GER); Simonet Roda, Maria (LMU Munich, Munich, GER); Ziegler, Andreas (University of Ulm, Ulm, GER); Fernandez-Diaz, Lurdes (Universidad Complutense de Madrid, Madrid, ESP); Schmahl, Wolfgang (LMU Munich, Munich, GER)

Most biocarbonates show a nanoparticulate morpho-topology in the 50-100 nm size range in high resolution imaging studies. Accordingly, a paradigm evolved claiming that biominerals form by accretion of carbonate-filled vesicles exocytosed by the mineralizing cells. We investigated numerous marine calciumcarbonate shells with AFM, SEM/EBSD, TEM and XRD over the last decade, and we likewise examined calcite crystals grown abiotically in a protein or polysaccharide gel matrix. A number of observations speak against nanoparticle accretion as a general model for biomineralization and for an ion-by-ion growth mechanism:

(1) Gel-grown calcite incorporates a fibrous gel network with connected pores in the 50-100 nm range. These crystals thus show a superficial "nanoparticulate" mesocrystal-like appearance where the 100 nm subcrystals result from the gel pores rather than from pre-existing nanoparticles. The crystals show $\{104\}$ morphologies which are created by ion-by-ion growth.

(2) For most marine biocarbonates the "nanogranule-like" morphologies are also in the 50-100 nm range, and the biocarbonate crystals also contain incorporated organic networks with pores in the corresponding size-range. One exception are coccoliths, which do not contain organic matrix and show a classical crystallography; moreover, THEY form in intracellular vesicles.

(3) Packing of any round similarly-sized particles leaves $25 \%$ pore space. However, most marine biocarbonates are rather dense.

(4) We showed for brachiopods that the shell mineral morphology is tightly controlled by the epithelial cell membranes and that "extrapallial space" is absent, which speaks for ion-by-ion growth. No vesicles supporting the paradigm of "nanoparticle-accretion" of the shell calcite were found. 